\title{
OÚRICURI
}

\section{ESTUDO ETNOBOTÂNICO DE PLANTAS MEDICINAIS DA MATA CILIAR DO SUBMÉDIO SÃO FRANCISCO, NORDESTE DO BRASIL}

\author{
Benedita Ramila Barbosa da SILVA ${ }^{1}$; Cecília de Fátima Castelo Branco Rangel de ALMEIDA ${ }^{2^{\star}}$ \\ ${ }^{1}$ Licenciada em Ciências Biológicas pelo Centro de Ensino Superior do Vale do São Francisco - \\ CESVASF. Mestranda em Biociências pela Universidade Federal do Vale do São Francisco - UNIVASF, \\ Laboratório de Farmacognosia e Fitoterapia - LFF. Avenida José de Sá Maniçoba, s/n- Centro, Petrolina / \\ PE - CEP: 56304-917. E-mail: benedita.ramila@hotmail.com. \\ Bacharel em Ciências Biológicas pela Universidade Federal de Pernambuco - UFPE. Doutorado em \\ Ciências Farmacêuticas pela Universidade Federal de Pernambuco - UFPE. Professora do Centro de \\ Ensino Superior do Vale do São Francisco - CESVASF. Rodovia BR-315, s/n - Alto do Encanto, Belém de \\ São Francisco / PE - CEP: 56440-000. *Autor correspondente. E-mail: ccastelobranco@yahoo.com.br. \\ Recebido: 22.09.2020 Aceito: 15.12.2020 \\ https://doi.org/10.29327/ouricuri.10.1-6
}

Resumo: A mata ciliar do Submédio São Francisco possui grande fitodiversidade com atividade biológica potencial e relação intrínseca com a população ribeirinha. Com isso, o objetivo desta pesquisa foi identificar espécies conhecidas e utilizadas como medicinais atestadas pela tradicionalidade do uso. Os sujeitos da pesquisa foram os moradores da comunidade de Jatubarana localizada a 600 metros das margens do Rio São Franciscono município de Chorrochó, Bahia. A coleta de dados ocorreu por pesquisa de campo, aplicando a lista livre e questionário estruturado, baseando-se no conceito de "chefe de família". Foi registrado um total de 59 espécies e 31 famílias botânicas, destas, cinco famílias contribuíram com um maior número de espécies: Fabaceae (9), Euphorbiaceae (6), Lamiaceae (4), Anacardiaceae (4), Apiaceae (4). Conforme a farmacopéia popular da comunidade estudada, as plantas citadas possuem eficácia no alivio da enfermidade caracterizando o potencial medicinal das espécies nativas e exóticas da Caatinga. O caule foi à parte da planta mais citada no uso terapêutico, à forma de preparo dos remédios mais utilizados foi o chá e a indicação terapêutica mais citada foi para doença respiratória. Doze espécies apresentaram grande Importância Relativa quanto aos usos medicinais com $\mathrm{IR} \geq 2$ indicados para até três sistemas corporais, a espécie mais versátil foi o Cymbopogon citratus (DC.) Staph com IR $(2,0)$. O estudo foi essencial para o resgate etnobotânico, identificando as plantas de interesse medicinal do bioma Caatinga.

Palavras-chave: Caatinga; Conhecimento Tradicional; Floresta Ciliar.

\section{ETHNOBOTANICAL STUDY OF MEDICINAL PLANTS FROM THE CILIARY FOREST OF THE SUBMEDIATE SÃO FRANCISCO, NORTHEAST OF BRAZIL}

\begin{abstract}
The Riparian Forest of Submediate São Francisco has great phytodiversity, with potential biological activity and intrinsic relationship with the riverside population. Thus, the objective of this research was to identify species known and used as medicinal attested by the traditionality of use. The research subjects were the residents of the community of Jatubarana located 600 meters from the banks of the São Francisco River in the municipality of Chorrochó, Bahia. Data collection occurred by field research, applying the free list and structured questionnaire, based on the concept of "head of family". A total of 59 species and 29 botanical families were recorded, of which five families contributed a greater number of species: Fabaceae (9), Euphorbiaceae (7), Lamiaceae (4), Anacardiaceae (4), Apiaceae (4). According to the popular pharmacopoeia of the studied community, the mentioned plants have efficacy in relieving the disease, characterizing the medicinal potential of the native and exotic species of the Caatinga.
\end{abstract}


The stem was apart from the most cited plant in the therapeutic use, the way of preparing the most used remedies was tea and the most cited therapeutic indication was for respiratory disease. Twelvespecies presented great Relative Importance regarding medicinal uses with $I R \geq 2$ indicated for up to three body systems, the most versatile species was Cymbopogon citratus (DC.) Staph with IR $(2,0)$. The study was essential for the ethnobotanical rescue, identifying the plants of medicinal interest of the Caatinga biome, raising awareness of the importance of the Riparian Forest and its conservation.

Keywords: Caatinga; Traditional knowledge; Riparian Forest.

\section{ESTUDIO ETNOBOTÁNICO DE PLANTAS MEDICINALES DEL BOSQUE RIPARIANO DEL SUBMÉDIO SAN FRANCISCO, NORESTE DE BRASIL}

Resumen: El Bosque Ripariano del submédio San Francisco tiene una gran fitodiversidad, com actividad biológica potencial y relación intrínseca com la población ribereña.Por lo tanto, el objetivo de esta investigación fue identificar espécies conocidas y utilizadas como medicinales atestiguadas por La tradicionalidad de uso. Los sujetos de investigación fueron los residentes de la comunidad de Jatubarana ubicada a 600 metros de las orillas del río San Francisco en el municipio de Chorrochó, Bahía. La recopilación de datos se produjo mediante investigaciones de campo, aplicando la lista gratuita y el cuestionario estructurado, basado en el concepto de "cabeza de familia". Se registraron un total de 59 especies y 29 familias botánicas, de lascuales cinco famílias contribuyeron con un mayor número de especies: Fabaceae (9), Euphorbiaceae (7), Lamiaceae (4), Anacardiaceae (4), Apiaceae (4). Según la farmacopea popular de la comunidad estudiada, las plantas mencionadas tienen eficacia para aliviar la enfermedad, caracterizando el potencial medicinal de las especies nativas y exóticas de la Caatinga. El tallo era aparte de la planta más citada en el uso terapéutico, la forma de preparar los remedios más utilizados era el té y la indicación terapéutica más citada era para las enfermedades respiratórias. Doce espécies mostraron una gran Importancia Relativa con respecto a los usos medicinales con IR $\geq 2$ indicado para hasta tres sistemas corporales, la especie más versátil fue Cymbopogon citratus (DC.) Staph con IR $(2,00)$. El estúdio fue esencial para el rescate etnobotánico, identificando las plantas de interés medicinal del bioma Caatinga, concienciando sobre la importância del Bosque Ripariano y su conservación.

Palabras clave: Caatinga; Conocimiento Tradicional; Bosque Ripariano.

\section{INTRODUÇÃO}

No final do século XIX a expressão "Etnobotânica" surgiu pela primeira vez no meio científico; estudiosos da área atribuem a autoria da expressão a John W. Harshberger, botânico norte americano (Schultes e Reis, 1995; Clément, 1998). Em 1896, a expressão surge em artigos científicos em que Harshberger disserta a criação de um museu com utensílios aborígenes, constando os objetivos principais da Etnobotânica (Harshberger, 1896; Clément, 1998). No ano que Harshberger publicou seu artigo foi publicado também o artigo - "A contribution to ethnobotany" já empregando a expressão Etnobotânica (Oliveira et al., 2009).

A Etnobotânica é definida como a "interação entre pessoas e plantas" (Albuquerque, 2005), o que inclui todas as formas de percepção e apropriação dos recursos vegetais (Albuquerque e Hanazaki, 2006). Pesquisas etnobotânicas contribuem para o resgate, registro, valorização do conhecimento popular, conhecimento científico das espécies, relação do homem 
com a natureza e conservação da biodiversidade local, tornando esses saberes acessíveis às futuras gerações, possibilitando a realização de novos estudos (Ribeiro, 2018).

A Caatinga apresenta uma grande variedade de plantas medicinais utilizadas na medicina popular pelas comunidades tradicionais (Alves et al., 2019), sendo uma importante fonte de novos compostos bioativos para o tratamento de doenças (Malafaia et al., 2017). Diversas espécies vegetacionais das matas ciliares da Caatinga possuem potencial para uso terapêutico, sendo utilizadas para a cura de enfermidades ou sintomalogias pelas populações locais, que geração a geração transmitem sua farmacopéia popular, com base nas experiências adquiridas, sobre o uso terapêutico das plantas.

A vegetação ciliar é considerada Área de Preservação Permanente pelo Código Florestal, (Lei no. 12.651, de 25 de maio de 2012), sendo uma área coberta ou não por floresta nativa, tendo à função ambiental de preservar os recursos hídricos, a diversidade biológica, a paisagem, a estabilidade geológica, e proporcionar o bem-estar das populações humanas (Brasil, 2012).

Todavia, as matas ciliares estão sendo degradadas em detrimento de atividade antrópica (Cunha, 2017) que vem ocorrendo na paisagem do Submédio São Francisco que ao longo dos anos vem passando por mudanças onde a vegetação nativa da Caatinga é gradativamente eliminada para uso extrativista e agrícola (Cunha, 2011).

Diversas propriedades terapêuticas de espécies da mata ciliar da Caatinga são desconhecidas pela comunidade científica, todavia, esse rico acervo botânico pode vir a se perder, caso o desmatamento nas margens de rios avance, sem uma eficaz proteção dos remanescentes e restauração de áreas atualmente desmatadas.

Tendo em vista que a recuperação da vegetação ripária no curso da Bacia do Rio São Francisco é fundamental para reverter o quadro de degradação atual (Ferreira et al., 2009), o estudo etnobotânico vem a desempenhar importantes funções, registrando as espécies de interesse medicinal, o que contribuirá para estudos conservacionistas e de bioprospecção (Albergaria et al., 2019). Com manejos sustentáveis, que se oponham as formas destrutivas atuais, conciliando preservação da biodiversidade de espécies florísticas da Caatinga com os povos que detêm o conhecimento sobre o uso das plantas.

O objetivo desta pesquisa foi identificar espécies conhecidas e utilizadas como medicinais atestadas pela tradicionalidade do uso.

\section{MATERIAL E MÉTODOS}

\section{Área de estudo}

A área de estudo foi à comunidade rural de Jatubarana localizada a 600 metros das margens do Rio São Francisco, com distância de 48 km da sede do município de Chorrochó, Bahia. A comunidade rural de Jatubarana foi fundada por agricultores e pescadores da região, com uma população estimada de 385 pessoas e 75 casas. Dispõe de uma escola e não possui 
posto médico, a comunidade apresenta "bancos de areia" nas margens do Rio São Francisco, formando uma praia de água doce, propicia ao banho, entretanto ainda não dispõe de estrutura para turismo.

A população vive da agricultura irrigada e pesca, sendo as principais atividades econômicas da localidade, as mulheres são as responsáveis pelo cuidado do lar e algumas são fazendeiras, possuem hortas nos quintais onde cultivam espécies vegetais para subsistência e comercialização. Os homens são os responsáveis pelas atividades agrícolas em suas propriedades, prestam serviços de curto prazo a outros agricultores e também são os responsáveis pela pesca.

O município de Chorrochó localiza-se na mesorregião do São Francisco (Figura 1), território de identidade: Itaparica/BA situa-se acerca de $450 \mathrm{~km}$ da capital Salvador/BA, com acesso pela BR-116. Segundo o Atlas dos Municípios de Abrangência do Centro de Ensino Superior do Vale do São Francisco (CESVASF) (Almeida e Silva, 2016), aponta uma área territorial de $3.005,318 \mathrm{~km}^{2}$ e uma estimativa de 11.522 habitantes com densidade demográfica 3,57 hab/ km², o Índice de Desenvolvimento Humano Municipal (IDHM) é de 0, 600 (IBGE, 2010).

Banhado pela bacia do Rio São Francisco, possuindo uma área de 638.32 km², o bioma característico é a Caatinga ocupando uma área de aproximadamente $844.453 \mathrm{~km}^{2}$, o que equivale a $11 \%$ do território brasileiro (BRASIL, 2014), internacionalmente vem sendo considerado como parte das Florestas Tropicais Sazonalmente Secas (Souza et al., 2015).

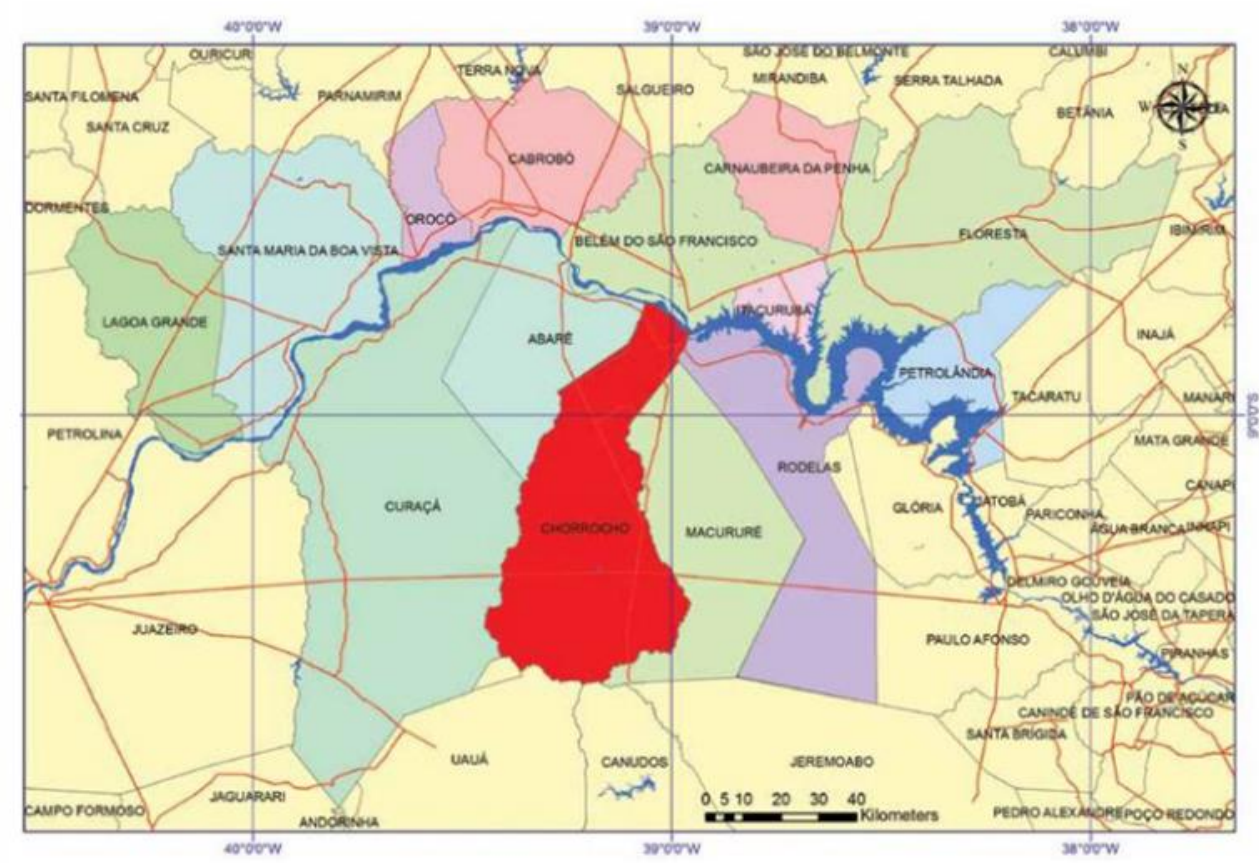

Figura 1. Mapa do município de Chorrochó/BA destacado em vermelho (Fonte: Almeida e Silva, 2016).

\section{Coleta de dados}


A coleta de dados ocorreu entre março e abril de 2019, sendo realizada por meio de pesquisa de campo e os dados obtidos através de lista livre e questionário estruturado com perguntas abertas e fechadas (Albuquerque e Lucena, 2004). A lista livre permite obter informações sobre um domínio cultural de uma comunidade, os informantes da pesquisa são solicitados a listar, como exemplo, as plantas medicinais conhecidas da região, sendo que elementos culturalmente importantes aparecerão com frequência em muitas listas com ordem de importância (Albuquerque et al., 2010). No questionário estruturado os informantes responderam as mesmas perguntas previamente estabelecida e na mesma sequência (Albuquerque e Lucena, 2004).

Anteriormente a entrevista ocorreu o cumprimento dos aspectos legais, como a assinatura do Termo de Consentimento Livre e Esclarecido, seguindo os aspectos éticos legais da Resolução 196/96 do Comitê de Ética em Pesquisa (Ministério da Saúde, 2002). Foram entrevistados 30 moradores, baseando-se no conceito de "chefe de família" em que apenas o adulto responsável pela residência e que detém o maior conhecimento sobre o assunto é entrevistado (Costa e Marinho, 2016).

Os informantes responderam perguntas sobre os dados socioeconômicos (nome, ano de nascimento, sexo, ocupação atual, grau de escolaridade, renda média familiar) e dados etnobotânicos (listar espécies de plantas medicinais da mata ciliar da Caatinga, indicações terapêuticas, modos de preparo, como obtém o recurso, eficácia no alivio da sintomalogia ou enfermidade, origem do conhecimento sobre plantas medicinais e utilização do recurso para consumo ou venda).

As entrevistas foram conduzidas de forma simples e com naturalidade devido à complexidade que envolve a Etnobotânica, a fim das respostas serem autênticas, evitaram-se por parte do pesquisador possíveis comentários que influenciassem as respostas dos entrevistados (Posey, 1986).

Os nomes populares das espécies florísticas nativas e exóticas foram registrados conforme citação dos informantes, os nomes científicos foram validados por consulta ao livro Sistemática Vegetal: Um enfoque filogenético (Judd et al., 2009) e consulta aos sites "The Plant List" (http://www.theplantlist.org) e "species Link" (http://www.splink.org.br).

\section{Análise de dados}

Os dados coletados foram tabulados e analisados quali-quantitativamente, com utilização do software Office Excel 2010 da Microsoft para a construção de tabelas, gráficose demais recursos fundamentais para a comprovação dos resultados atingidos no decorrer da pesquisa.

Foi calculado o índice de Importância Relativa (IR) das espécies medicinais, conforme a fórmula: $\mathrm{IR}=\mathrm{NSC}+\mathrm{NP}$, onde $\mathrm{NSC}=$ Número de Sistemas Corporais tratados por cada espécie (NSCE) dividido pelo Número total de Sistemas Corporais tratados pela espécie mais Versátil 
(NSCEV); NP = Número de Propriedades Atribuídas a uma determinada Espécie (NPE) dividido pelo Número total de Propriedades Atribuídas à Espécie mais Versátil (NPEV) (Almeida e Albuquerque, 2002).O valor máximo da IR atribuída a cada planta será igual a 2.

\section{RESULTADOS E DISCUSSÃO}

\section{Perfil socioeconômico}

Dos 30 entrevistados $87 \%$ eram do sexo feminino e $13 \%$ do sexo masculino. A maior parcela foi de mulheres, visto que, o sexo feminino detém um conhecimento maior sobre o tema, dominam a manipulação e uso de plantas medicinais, além de serem conhecedoras da farmacopeia popular de suas localidades e se responsabilizarem mais nos cuidados com a saúde da família (Leite et al., 2015; Ferreira et al., 2016; Oliveira, Oliveira e Andrade, 2010).

A faixa etária dos entrevistados variou dos 20 aos 68 anos (Figura 2), considerando este intervalo de idades, as faixas mais frequentes possuem idade superior aos 40 anos $(70 \%$ dos entrevistados). Indicando que indivíduos de idade mais avançada possuem em geral maiores conhecimentos sobre plantas medicinais (Fagundes, Oliveira e Souza, 2017; Neto e Gomes, 2018), entre a população jovem as práticas sobre o uso medicinal de plantas vêm se perdendo, devido a degradação ambiental e perda de transmissão cultural entre as gerações (Fagundes, Oliveira e Souza, 2017).

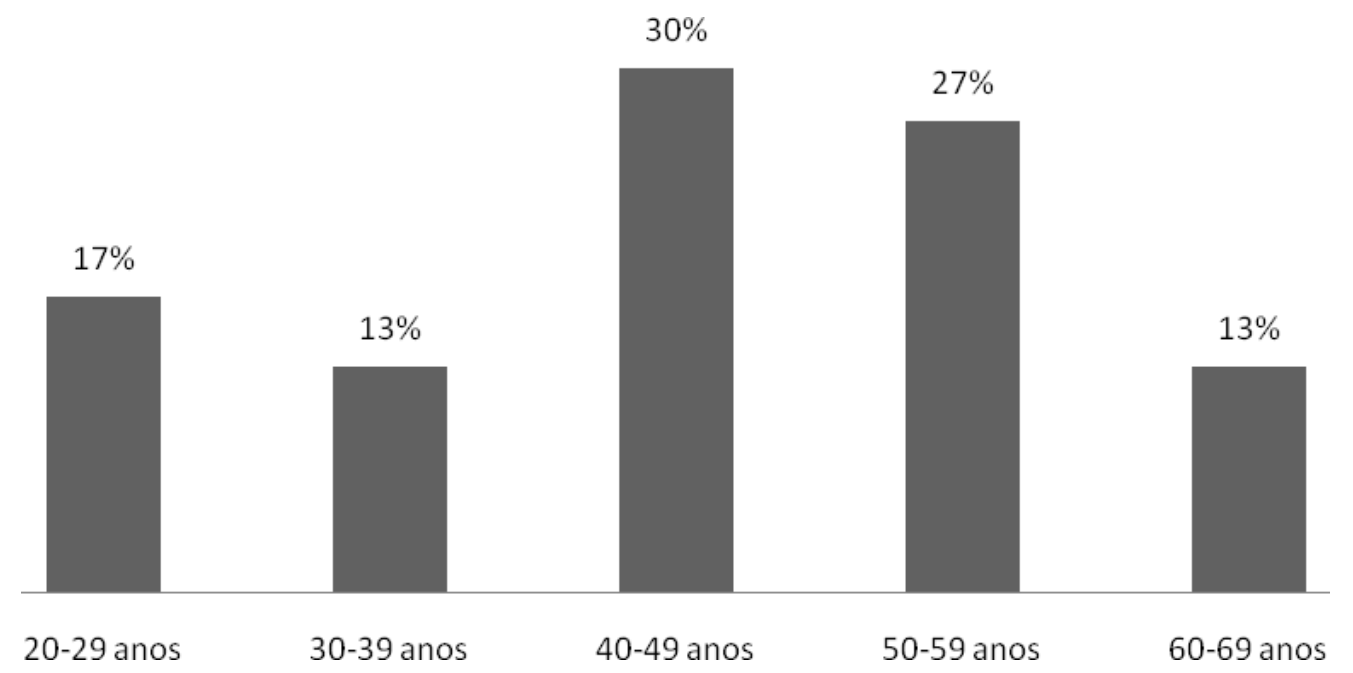

Figura 2. Faixa etária dos entrevistados na comunidade de Jatubarana, Chorrochó/BA no Submédio do Rio São Francisco.

Quanto à escolaridade dos entrevistados, $10 \%$ são analfabetos, $50 \%$ possuem Ensino Fundamental Incompleto, 17\% têm Ensino Fundamental Completo, 10\% Ensino Médio Incompleto, 10\% detêm Ensino Médio Completo e apenas 3\% afirmaram possuir Ensino Superior Completo. Este resultado indica que o conhecimento e uso de plantas medicinais concentra-se entre os que possuem no máximo o Ensino Fundamental Completo, é citado na literatura que 
níveis baixos de escolaridade têm sido positivamente relacionados a um maior conhecimento e uso das plantas nativas (Albuquerque et al., 2011; Pereira et al., 2015; Soares et al., 2015;Gaoue et al., 2017).

Os dados referentes à renda familiar demonstram que $98 \%$ dos entrevistados se encaixam na menor faixa de renda. Inferindo-se que a maior familiarização com as espécies medicinais reflete a busca por formas alternativas de tratar doenças (Neto e Gomes, 2018).

\section{Levantamento etnobotânico}

Foi registrado um total de 59 espécies de plantas, conhecidas e utilizadas pela população entrevistada, pertencentes a 53 gêneros e 31 famílias botânicas (Tabela 1). Destas, cinco famílias contribuíram com um maior número de espécies: Fabaceae (9), Euphorbiaceae (6), Lamiaceae (4), Anacardiaceae (4) e Apiaceae (4). Em muitos trabalhos a família Fabaceae também se destaca (Da Silva, 2012; Costa e Marinho, 2016; Bastos et al., 2018). Este resultado deve-se a ampla distribuição desta família e alta representatividade em regiões tropicais secas.

As plantas citadas tem uma extensa finalidade terapeutica, sendo utilizadas para combater doenças respiratórias, infecção, problemas gastrointestinais, cálculos renais, vermes e nervosismo. Com maior número de citações para doenças respiratórias. No estudo realizado por Ruzza et al. (2014) e Merly e Santos (2017) as doenças respiratórias também se destacaram.

A maioria das espécies vegetais citadas é de origem exótica representando $59 \%$ e $41 \%$ de origem nativa, demonstrando que as espécies nativas da mata ripária da Caatinga estão reduzidas a áreas remanescentes. As espécies exóticas predominam, pois são mais competitivas frente às espécies nativas, nos ambientes que colonizam, formando populações adensadas que ocupam grandes áreas, ao se estabelecerem em novas áreas, pode não haver predadores dessas espécies exóticas, propiciando uma maior densidade populacional, com efeitos negativos sobre as espécies nativas, alterando a composição e estrutura fisionômica de uma comunidade biológica (Martins, 2004).

O caule foi à parte da planta mais indicada pelos informantes no uso terapêutico (Figura 3), (52\% das citações). Este resultado, corrobora os trabalhos de Oliveira e Lucena (2015), Gois (2016), que indicavam um maior número de citações para as folhas no uso medicinal. O caule é extraído geralmente das espécies nativas (Cordeiro e Felix, 2014; Macêdo et al., 2015).

O uso do caule das plantas da Caatinga justifica-se pela disponibilidade deste durante todo o ano, inclusive durante a estação seca, o que não ocorre com as folhas, flores e frutos (Albuquerque e Andrade, 2002; Cartaxo et al., 2010). Em seguida se sobressaiu às folhas com $29 \%$ das citações, de maior parte de plantas exóticas, o uso das folhas deve-se, provavelmente, a utilização de plantas medicinais herbáceas, pelas pessoas da comunidade. 
Tabela 1. Espécies de plantas identificadas de uso medicinal, pelos entrevistados da comunidade de Jatubarana, Chorrochó/BA no Submédio do Rio São Francisco. N= nativa, E= exótica.

FAMÍLIA/ESPÉCIE

NOME POPULAR

INDICAÇÃO

ORIGEM

\section{AMARANTHACEAE}

1. Celosia argentea L.

2. Dysphania ambrosioides L.

ANACARDIACEAE

3. Anacardium occidentale L.

4. Myracrodruon urundeuva

Allemão

5. Schinopsis brasiliensis Engl.

6. Spondias tuberosa Arruda

\section{ANNONACEAE}

7. Annona muricata $\mathrm{L}$.

APIACEAE

8. Coriandrum sativum $\mathrm{L}$.

9. Foeniculum vulgare Mill.

10. Petroselinum crispum (Mill.)

Fuss

11. Pimpinella anisum $\mathrm{L}$.

\section{APOCYNACEAE}

12. Aspidosperma pyrifolium Mart.

ASTERACEAE

13. Egletes viscosa (L.) Less.

14. Matricaria chamomilla L.

BIGNONIACEAE

15. Tabebuia sp.

16. Tabebuia aurea (Silva Manso)

Benth. \& Hook.f. ex S. Moore

BRASSICACEA

17. Nasturtium officinale $\mathrm{R}$. $\mathrm{Br}$.

CACTACEAE

18. Cereus jamacaru DC.

CARYOPHYLLACEAE

19. Dianthus caryophyllus L.

CELASTRACEAE

20. Maytenus rigida Mart.

CUCURBITACEAE

21. Citrullus lanatus (Thunb.)

Matsum \& Nakai

\section{EUPHORBIACEAE}

22. Cnidoscolus quercifolius Pohl

23. Cnidoscolus urens (L.) Arthur

24. Croton blanchetianus Baill.

25. Croton campestris A.St.-Hil.

26. Mallotusrhamnifolius H.B.K.

27. Ricinus communis $\mathrm{L}$.

FABACEAE

28. Amburana cearensis (Allemão)

A. C. Sm.

29. Anadenanthera colubrina (Vell)

Brenan

30. Bauhinia forficata Link.

31. Caesalpinia ferrea C. Mart.

32. Caesalpinia pyramidalis Tul.
Crista-de-galo

Mastruz

Cajueiro

Aroeira

Baraúna

Umbuzeiro

Graviola

Coentro

Endro

Salsinha

Erva-doce

Pereiro

Marcela

Camomila

Pau-darco

Caraibeira

Agrião

Mandacaru

Cravo

Pau-de-colher

Melancia

Faveleira

Cansanção

Marmeleiro

Velame

Quebra-faca

Mamona

Umburana

Angico

Pata-de-vaca

Pau-ferro

Catingueira
Infecção qualquer tipo

Vermes

E

Infecção qualquer tipo

Inflamação vaginal, cicatrização de

parto, infecção urinária

Inflamação qualquer tipo, dor de

pancada

Infecção externa, infecção interna,

dor de pancada

Infecção interna, febre

E

Dor abdominal

Dor de cabeça

Inflamação na próstata

Enjôos

E

N

$\mathrm{N}$

$\mathrm{N}$

N

Dor no estômago

Nervosismo

Nervosismo

$\mathrm{E}$

Gripe

Hipertensão

$\mathrm{N}$

$\mathrm{N}$

E

N

E

N

E

Infecção externa N

Problema na coluna N

Gripe, gastrite N

Infecção interna N

Dor no estômago N

Vermes, câncer E

Cálculo renal, dor muscular, $\quad \mathrm{N}$

infecção externa

Gripe N

Diabetes $\quad E$

Gripe N

Gripe, dor no estômago N 
Tabela 1. Continuação.

FAMÍLIA/ESPÉCIE

NOME POPULAR

INDICAÇÃO

ORIGEM

\section{FABACEAE}

33. Hymenaea courbaril L.

34. Pithecellobium diversifolium

Benth.

35. Prosopis juliflora (Sw.) DC.

36. Tamarindus indicus $L$.

JUNCACEAE

37. JunCus SP

LAMIACEAE

38. Mentha spicata $\mathrm{L}$.

39. Ocimum brasilicum L.

40. Ocimum gratissimum Lam.

41. Plectranthus barbatus Andrews

LECYTHIDACEAE

42. Eschweilera ovata (Cambess.)

Mart. ex Miers

LYTHRACEAE

43. Punica granatum $\mathrm{L}$.

MORACEAE

44. Morus nigra L.

MYRTACEAE

45. Eucalyptus grandis W. Hill

OLACACEAE

46. Ximenia americana $\mathrm{L}$.

PASSIFLORACEAE

47. Passiflora sp.

PHYLLANTACEAE

48. Phyllanthus niruri L.

POACEAE

49. Cymbopogon citratus (DC.)

Staph

50. Saccharum officinarum L.

RUTACEAE

51. Citrus $\times$ aurantium L.

52. Citrus limon $\mathrm{L}$.

53. Ruta graveolens $\mathrm{L}$.

RUBIACEAE

54. Uncaria tomentosa (Willd. ex

Roem. \& Schult.) DC

RHAMNACEAE

55. Zizyphus joazeiro Mart.

SOLANACEAE

56. Solanum melongena $\mathrm{L}$.

SAPOTACEAE

57. Sideroxylon obtusifolium

(Roem. \& Schult.) T.D.Penn.
Jatobá

Gripe

Carcarazeiro

Gripe

Gripe

Algaroba

Tamarindo

Diabetes, vermes

Junco

Gripe

Gripe

Hortelã

Manjericão

Alfavaca

Malva-santa

Embiruba

Romã

Gripe

Inflamação interna

Gripe

Cálculo renal

E

Amora Cálculo renal E

Eucalipto

Tosse, febre

E

Ameixa

Maracujá

Infecção interna

$\mathrm{N}$

$\mathrm{E}$

Quebra pedra

Nervosismo

Cálculo renal

$\mathrm{N}$

Capim-santo

Cana-de-açúcar

Falta de apetite, má digestão, dor de cabeça, tosse

Hipertensão

E

E

Laranjeira $\quad$ Febre, Gripe

Limoeiro

Arruda

Gripe

Dores musculares

Cisto no ovário

E

Juazeiro

Gripe

$\mathrm{N}$

Berinjela

Colesterol alto

E

Quixabeira

Problemas na coluna

$\mathrm{N}$

Erva-cidreira

Gripe

E

Babosa

Gripe

E

N

E

E

E

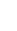

E

$N$

E

E

E

E

N
$\mathrm{N}$

E

E

E

E

(1)

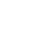

E

E

E

E

$N$

58. Lippia alba (Mill.) NEBr. ex

Britton \& P.Wilson

XANTHORRHOEACEAE

59. Aloe vera (L.) Burm. f. 


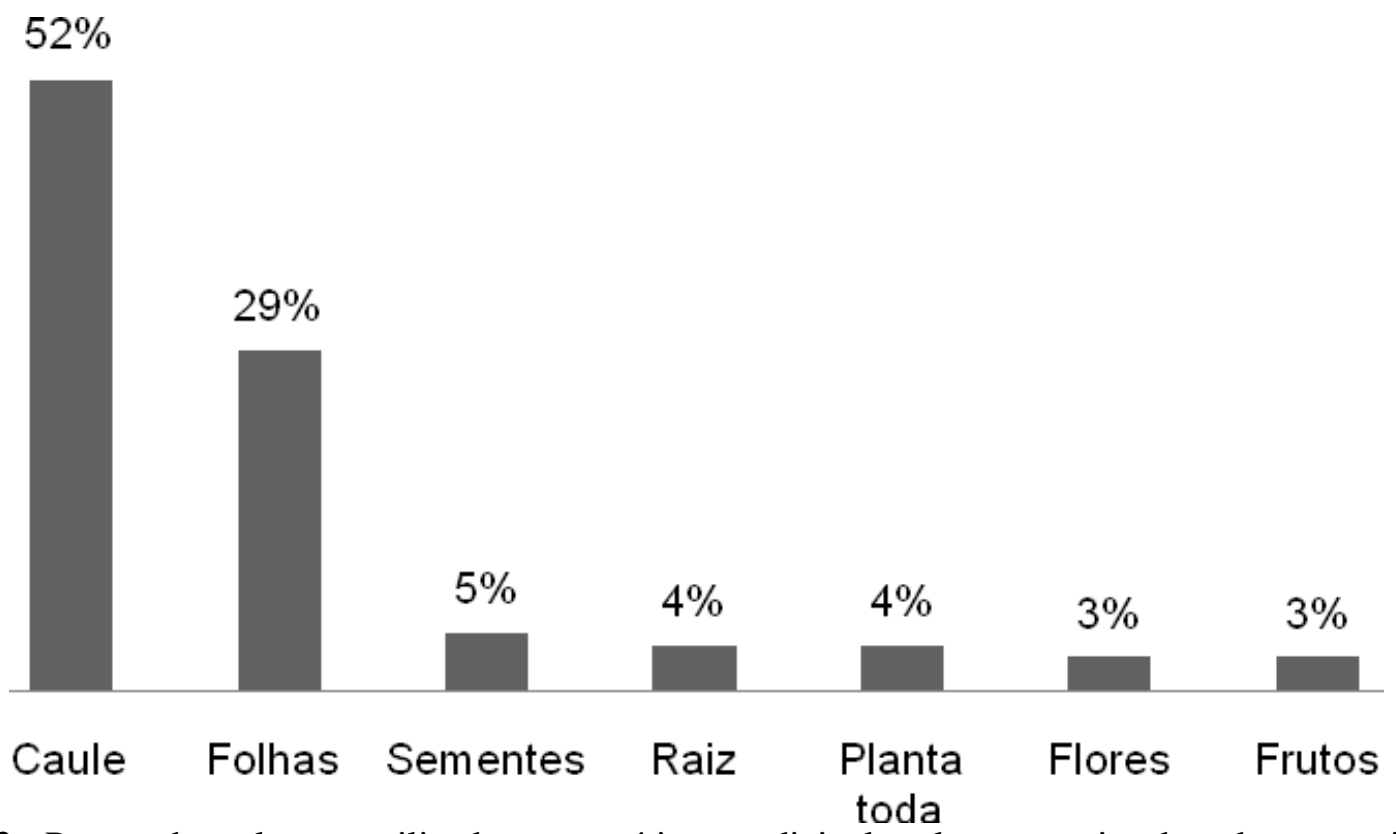

Figura 3. Partes das plantas utilizadas na prática medicinal pelos entrevistados da comunidade de Jatubarana, Chorrochó/BA no Submédio do Rio São Francisco.

A forma de preparo dos remédios mais utilizados foi o chá (infusão e decocção), representando $42 \%$ das citações (Figura 4), tradicionalmente os chás são preparações terapêuticas muito utilizadas na cura e prevenção de agravos à saúde. Para infusão é indicado utilizar as partes tenras como folhas, flores e frutos, para decocção utiliza-se partes mais duras como raízes, rizomas, cascas, caules e sementes (Dluzniewski, Vettorato e Müller, 2018).

Doze espécies demonstraram grande importância relativa (IR) quanto aos usos medicinais, com IR $>1$, indicadas para até três sistemas corporais, tendo assim importância para comunidade devido a sua versatilidade terapêutica, as demais, 47 espécies, evidenciaram um IR variando de 0,58 a 0,83 (Tabela 2).

A espécie mais versátil com maior IR foi o $C$. citratus - IR 2,0, seguido por $A$. cearenses IR 1,75, M. urundeuva - IR 1,41 e $S$. tuberosa - IR 1,41. A espécie $C$. citratus, apresentou em estudos farmacológicos, atividade diurética, antinociceptivo, ação antioxidante, antifúngica e antihelmíntico (Almeida e Santos, 2018), confirmando sua versatilidade terapêutica, condizente com algumas informações obtidas na comunidade sobre o uso desta planta. 


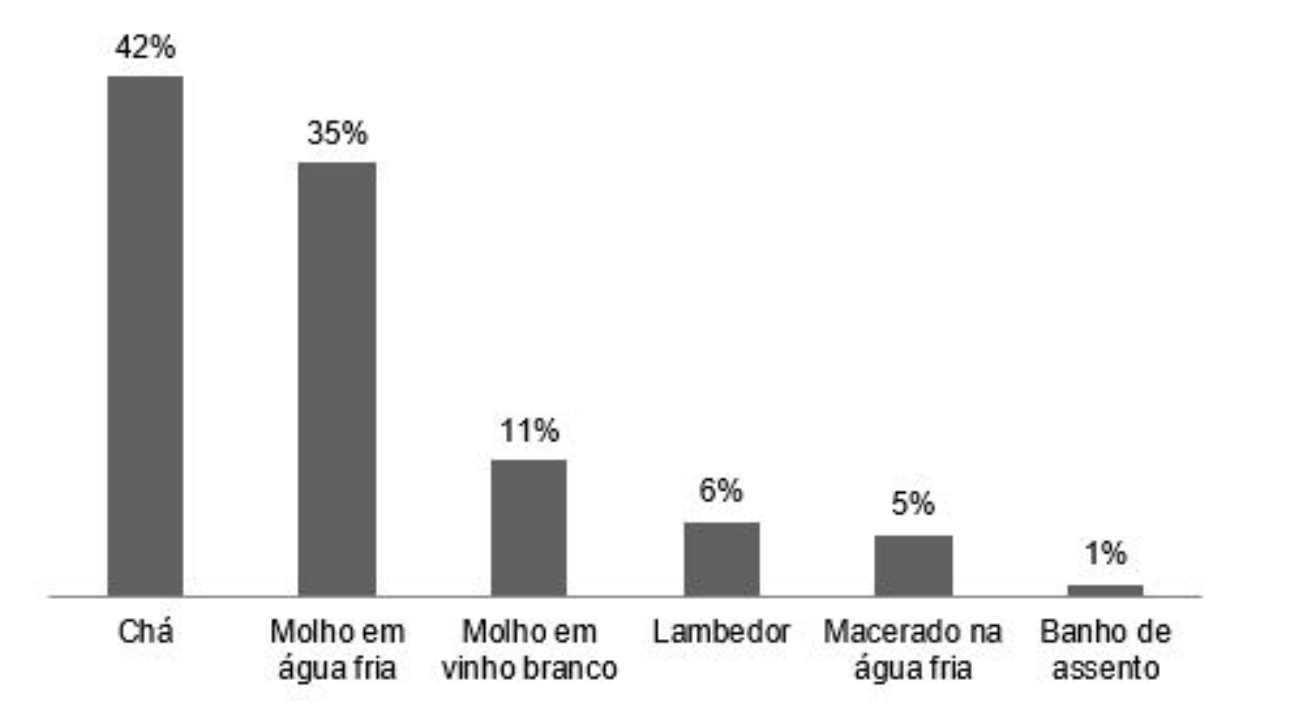

Figura 4. Modos de preparo citados pelos informantes da comunidade de Jatubarana, Chorrochó/BA no Submédio do Rio São Francisco.

Entre os entrevistados $90 \%$ informaram que coletam as plantas medicinais na mata, $7 \%$ asseguraram que cultivam e $3 \%$ afirmaram que compram as espécies medicinais. Isso demonstra uma dependência das matas por parte dos moradores da comunidade, e o quanto é fundamental que a população preserve este recurso.

Neste estudo foi apontado que 100\% dosinformantes afirmaram que as plantas citadas possuem eficácia no alívio de sintomalogia ou enfermidade. O que caracteriza o potencial medicinal das espécies nativas e exóticas da Caatinga, com base no conhecimento tradicional da população que geração a geração constroem sua farmacopéia popular através das experiências sob o uso terapêutico das plantas.

O conhecimento sobre plantas medicinais tem origem familiar representando $97 \%$ das citações e 3\% adquiriram o conhecimento na feira. O conhecimento tradicional está sendo repassado para as gerações seguintes através da oralidade, os informantes afirmaram que adquiriram os conhecimentos sobre plantas medicinais com a avó, mãe ou parente mais velho. Portanto, os conhecimentos são adquiridos na própria localidade onde residem, demonstrando um rico patrimônio cultural sobre conhecimentos etnomedicinais, visto isso, esta pesquisa torna-se um documento que contribui para a preservação deste patrimônio e uso de recursos botânicos medicinais.

As espécies vegetais são utilizadas para consumo próprio, representando $98 \%$ das citações e apenas $2 \%$ informaram que utilizam para venda, indicando que as pessoas usam 0 recurso e vendem apenas o excedente como uma forma complementar da renda familiar. 
Tabela 2. Valores da Importância Relativa (IR) de cada espécie de planta medicinal conhecida pelos informantes da comunidade de Jatubarana, Chorrochó/BA no Submédio do Rio São Francisco.

\begin{tabular}{|c|c|c|c|}
\hline ESPÉCIES & IR & ESPÉCIES & IR \\
\hline Cymbopogon citratus & 2,00 & Eschweilera ovata & 0,58 \\
\hline Amburana cearensisSm. & 1,75 & Foeniculum vulgare & 0,58 \\
\hline Myracrodruon urundeuva & 1,41 & Hymenaea courbaril & 0,58 \\
\hline Spondias tuberosa & 1,41 & Juncus sp. & 0,58 \\
\hline Caesalpinia pyramidalis & 1,16 & Lippia alba & 0,58 \\
\hline Cereus jamacaru & 1,16 & Matricaria chamomilla & 0,58 \\
\hline Citrus $\times$ aurantium & 1,16 & Maytenus rigida & 0,58 \\
\hline Croton blanchetianus & 1,16 & Mentha spicata & 0,58 \\
\hline Eucalyptus grandis & 1,16 & Morus nigra & 0,58 \\
\hline Ricinus communis & 1,16 & Nasturtium officinale & 0,58 \\
\hline Schinopsis brasiliensis & 1,16 & Ocimum brasilicum & 0,58 \\
\hline Tamarindus indicus & 1,16 & Ocimum gratissimum & 0,58 \\
\hline Annona muricata & 0,83 & Passiflora sp. & 0,58 \\
\hline Aloe vera & 0,58 & Petroselinum crispum & 0,58 \\
\hline Anacardium occidentale & 0,58 & Phyllanthus niruri & 0,58 \\
\hline Anadenanthera colubrina & 0,58 & Pimpinella anisum & 0,58 \\
\hline Aspidosperma pyrifolium & 0,58 & Pithecellobium diversifolium & 0,58 \\
\hline Bauhinia forficata & 0,58 & Plectranthus barbatus & 0,58 \\
\hline Caesalpinia ferrea & 0,58 & Prosopis juliflora & 0,58 \\
\hline Celosia argentea & 0,58 & Punica granatum & 0,58 \\
\hline Citrus limon & 0,58 & Ruta graveolens & 0,58 \\
\hline Citrullus lanatus & 0,58 & Saccharum officinarum & 0,58 \\
\hline Dysphania ambrosioides & 0,58 & Sideroxylon obtusifolium & 0,58 \\
\hline Cnidoscolus quercifolius & 0,58 & Solanum melongena & 0,58 \\
\hline Cnidoscolus urens & 0,58 & Tabebuia sp. & 0,58 \\
\hline Coriandrum sativum & 0,58 & Tabebuia aurea & 0,58 \\
\hline Croton campestris & 0,58 & Uncaria tomentosa & 0,58 \\
\hline Dianthus caryophyllus & 0,58 & Ximenia americana & 0,58 \\
\hline Egletes viscosa & 0,58 & Zizyphus joazeiro & 0,58 \\
\hline Mallotus rhamnifolius & 0,58 & & \\
\hline
\end{tabular}




\section{CONCLUSÕES}

Identificou-se uma grande diversidade de plantas medicinais que são conhecidas e utilizadas, as plantas citadas possuem atividade biológica potencial conforme a farmacopéia popular da comunidade estudada, que geração a geração adquiriu conhecimentos através da experiência do uso, caracterizando o potencial medicinal das espécies nativas e exóticas da Caatinga.

As matas ciliares ao longo dos anos são alvo de degradação, essas áreas são preferidas para agricultura em decorrência dos solos férteis, serem próximas do recurso hídrico, local para construção de áreas de lazer, urbanização e empreendimentos imobiliários, visto isso, os pesquisadores e o poder público vêm despertando interesse para recuperação dessas áreas ripárias ao longo do Rio São Francisco com o propósito de reverter o quadro de degradação atual.

Esta pesquisa contribuirá para trabalhos futuros na área de Etnobotânica e Etnofarmacologia, além do que poderá subsidiar fundamentação teórica para um possível Plano de Conservação das Matas Ciliares da Caatinga. E a sistematização dos conhecimentos etnomedicinais propicia a conservação da diversidade florística e impacta na manutenção do acervo cultural da comunidade tradicional.

\section{AGRADECIMENTOS}

A CAPES pelo suporte financeiro à Benedita Ramila Barbosa da Silva. Aos moradores da comunidade rural de Jatubarana pela confiança e solicitude, por compartilharem seus conhecimentos, permitido assim concretizar esta pesquisa.

\section{REFERÊNCIAS}

Albergaria, E. T.; Silva, M. V.; Silva, A. G. Levantamento etnobotânico de plantas medicinais em comunidades rurais localizadas na Unidade de Conservação Tatu-Bola, município de Lagoa Grande, PE - Brasil. Revista Fitos, 13(2), 1808-9569, 2019.

Albuquerque, U.P, Andrade, L.H.C. Conhecimento botânico tradicional e conservação em uma área de caatinga no estado de Pernambuco, Nordeste do Brasil. Acta Botanica Brasilica. 16, 1677941X, 2002.

Albuquerque, U. P.; Lucena, R. F. P. Métodos e técnicas para coleta de dados In: Albuquerque, U. P. \& Lucena, R. F. P. (Org.). Métodos e Técnicas na pesquisa etnobotânica. Livro Rápido, Recife, 2004, p.37-62.

Albuquerque, U. P. Introdução à etnobotânica. 2. ed. Rio de Janeiro: Interciência, 2005.

Albuquerque, U. P.; Hanazaki, N. As pesquisas etnodirigidas na descoberta de novos fármacos de interesse médico e farmacêutico: fragilidades e perspectivas. Revista Brasileira de Farmacognosia, 16 (supl.), 0102-695X, 2006.

Albuquerque, U. P.; Lucena, R. F. P.; Alencar, N. L. Métodos e técnicas para coleta de dados etnobiológicos. In: Albuquerque, U. P.; Lucena, R. F. P.; Cunha, L. V. F. C. (Eds.) Métodos e técnicas na pesquisa etnobiológica e etnoecológica. Recife: NUPEEA, 2010, p.39-64. 
Albuquerque, U.P.; Soldati, G.T.; Sieber, S.S.; Ramos, M.A.; Sá, J.C.; Souza, L.C. The use of plants in the medical system of the Fulni-ô people (NE Brazil): A perspective on age and gender. Journal of Ethnopharmacology 133, 866- 873, 2011.

Almeida, C. F. C. B. R.; Albuquerque, U. P. Uso e conservação de plantas e animais medicinais no estado de Pernambuco (Nordeste do Brasil): um estudo de caso. Interciência, 27(6) 0378-1844, 2002.

Almeida, A. T.; Santos, A. F. Prospecção fitoquímica do extrato metanólico das folhas da Espécie Cymbopogon citratus. Diversitas Journal, 3(2) 2525-5215, 2018.

Almeida, C. F. C. B. R.; Silva, A. M. N. (Org.) Atlas dos municípios de abrangência do CESVASF: um resgate geográfico, histórico e biológico. Canal 6; Recife, PE: NUPEAA, 2016, p.192.

Alves, M. S.; Medeiros, M. A. A.; Pereira, C. T.; Simão, K. L. A.; Simão, B. L. A.; Filho, A. A. O. Avaliação da atividade antineoplásica e antiviral do monoterpeno Ascaridol presente em plantas da caatinga: estudo in silico. Revista Brasileira de Gestão Ambiental, 13(03), 2317-3122, 2019.

Bastos, E. M.; Silva, M. E. C.; Vieira, F. J.; Barros, R. F. M. Conhecimento botânico local em uma área de assentamento rural no Piauí, nordeste do Brasil. Gaia Scientia, 12(2),1981-1268, 2018.

Brasil. Lei n.12.651, de 25 de maio de 2012. Dispõe sobre a proteção da vegetação nativa; altera as leis 6.938, de 31 de agosto de 1981, 9.393, de 19 de dezembro de 1996, e 11.428, de 22 de dezembro de 2006; revoga as Leis 4.771, de 15 de setembro de 1965, e de 7,754, de 14 de abril de 1989, e a Medida Provisória no 2.166-67, de 24 de agosto de 2001; e dá outras providências, 2012. Diário oficial da União, Brasília, DF, Ano CXLIX, n.102, 28 maio 2012. Seção 1, p.1. Disponível em: https://www.google.com.br/url?sa=t\&source=web\&rct=j\&url=https://www2.camara.leg.br/legin/fed/l ei/2012/lei-12651-25. Acesso em: 11 de março de 2019.

Brasil. Ministério do Meio Ambiente. Bioma Caatinga: contexto, características e estratégias de conservação.2014. Disponível em: http://www.mma.gov.br/biomas/caatinga/item/191. Acesso em: 04 de março de 2019.

Cartaxo, S.L.; Souza, M.M.A.; Albuquerque, U.P. Medicinal plants with bioprospecting potential used in semi-arid northeast ern Brazil. Journal of Ethnopharmacology. 131, 0378-8741, 2010.

Clément, D. The historical foundations of ethnobiology (1860-1899). Journal of Ethnobiology, 18(2), 161-187, 1998.

Cordeiro, J. M. P.; Felix, L. P. Conhecimento botânico medicinal sobre espécies vegetais nativas da Caatinga e plantas espontâneas no agreste da Paraíba, Brasil. Revista Brasileira de Plantas Medicinais, 16(3), 1516-0572, 2014.

Costa, J. C.; Marinho, M. G. V. Etnobotânica de plantas medicinais em duas comunidades do município de Picuí, Paraíba, Brasil. Revista Brasileira de Plantas Medicinais, 18(1), 1516-0572, 2016.

Cunha, T. J. F.; Sá, I. B.; Neto, M. B. O.; Taura, T. A.; Filho, J. C. A.; Giongo, V.; Silva, M. S. L.; Drumond, A. Uso Atual e Quantificação de Áreas Degradadas na Margem Direita do Rio São Francisco no Município de Curaçá-BA. Revista Brasileira de Geografia Física, 4(6), 1197-1212, 2011.

Cunha, S. S. Conhecimento ecológico local sobre a mudança e conservação da mata ciliar do rio Taperoá, Paraíba: perspectivas para conservação. 27f. Monografia (Especialização em Etnobiologia) - Universidade Estadual da Paraíba, Campina Grande. 2017. 
Dluzniewski, F.S.; Vettorato, J.G.; Müller, N.T.G. Abordagem etnobotânica de Myrtaceae no município de Sete de Setembro, Rio Grande do Sul, Brasil. Revista Interdisciplinar em Ciências da Saúde e Biológicas, 2(1) 2594-7877, 2018.

Da Silva, C.G. Estudo etnobotânico e da atividade antimicrobiana "in vitro" de plantas medicinais na comunidade do Sítio Nazaré, município de Milagres. 93p. Dissertação (Mestrado em Ciências Florestais) - Universidade Federal de Campina Grande, UFCG, Patos. 2012.

Fagundes, N.C.A.; Oliveira, G.L.; Souza, B.G. Etnobotânica de plantas medicinais utilizadas no distrito de Vista Alegre, Claro dos Poções - Minas Gerais. Revista Fitos, 11(1), 2446-4775, 2017.

Ferreira, L.B.; Rodrigues, M.O.; Costa. J.M. Etnobotânica das Plantas Medicinais Cultivadas nos Quintais do Bairro de Algodoal em Abaetetuba/PA. Revista Fitos,10(3), 2446-4775, 2016.

Ferreira, R. A.; Santos, P. L.; Aragão, A. G.; Santos, T. I. S.; Neto, E. M. S.; Rezende, A. M. S. Semeadura direta com espécies florestais na implantação de mata ciliar no Baixo São Francisco em Sergipe. Scientia Forestalis, 37(81), 1413-9324, 2009.

Gaoue, O.G.; Coe, M.A.; Bond, M.; Seyler, B.C.; Mcmillen. 2017. Theories and Major Hypotheses in Ethnobotany. Economic Botany 71, 269-287, 2017.

Gois, M.A.F.; Lucas, F.C. A.; Costa, J.C.M.; Moura, P.H.B.; Lobato, G.J.M. Etnobotânica de espécies vegetais medicinais no tratamento de transtornos do sistema gastrointestinal. Revista Brasileira de Plantas Medicinais. 18(2), 1516-0572, 2015.

Harshberger, J. W. Purposes of ethnobotany. Botanical Gazette. 21, 00068071, 1896.

Instituto Brasileiro de Geografia e Estatística - IBGE. Censo 2010. Disponível em: http://www.ibge.gov.br. Acesso: 03 de março de 2019.

Judd, W.S.; Campbell, C.S.; Kellogg, E.A.; Stevens, P.F.; Donoghue, M.J. Sistemática Vegetal: Um Enfoque Filogenético. 3. ed. Porto Alegre: Artmed, 2009. 612p.

Leite, I. A.; Morais, A. M.; Ó, K. D. S.; Carneiro, R. G.; Leite, C. A. A etnobotânica de plantas medicinais no município de São José de Espinharas, Paraíba, Brasil. Biodiversidade, 14(1) 21771332, 2015.

Macêdo, D. G.; Ribeiro, D.A.; Coutinho, H.D.M.; Menezes, I.R.; Souza, M.M.A. Práticas terapêuticas tradicionais: uso e conhecimento de plantas do cerrado no estado de Pernambuco (Nordeste do Brasil). Boletín Latinoamericano y del Caribe de Plantas Medicinales y Aromáticas. 14(6) 717 7917, 2015.

Malafaia, C. B.; Jardelino, A. C. S.; Silva, A. G.; Souza, E. B.; Macedo, A. J.; Correia, M. T. S.; Silva, M. V. Effects of Caatinga Plant Extracts in Planktonic Growth and Biofilm Formation in Ralstonia solanacearum. Microbial Ecology, 75(3) 0095-3628, 2017.

Martins, S.V. Recuperação de Matas Ciliares. Viçosa, MG: Aprenda Fácil Editora, 2004, p.255.

Merhy, T.S.M.; Santos, M.G. A Etnobotânica na escola: interagindo saberes no ensino fundamental. Revista Práxis, (9)17, 2176-9230. 2017.

Ministério da Saúde, Conselho Nacional de Saúde. Manual Operacional para comitês de ética em pesquisa, Ministério da Saúde/Série CNS Cadernos Técnicos, 2002.

Neto, L. A. G.; Gomes, F. T. L. Levantamento etnobotânico de plantas medicinais utilizadas pela população do município de Oliveira Fortes - MG. Perspectivas Online: Biológicas \& Saúde. 8(27), 2236-8868, 2018. 
Oliveira, F. C.; Albuquerque, U. P.; Fonseca- Kruel, V. S.; Hanazaki, N. Avanços nas pesquisas etnobotânicas no Brasil. Acta Botânica Brasílica, 23(2), 1677-941X, 2009.

Oliveira, G.L.; Oliveira, A.F.M.; Andrade, L.H.C. Plantas medicinais utilizadas na comunidade urbana de Muribeca, Nordeste do Brasil. Acta Botanica Brasilica, 24(2), 1677-941X, 2010.

Oliveira, D.M.S.; Lucena, E.M.P. O uso de plantas medicinais por moradores de Quixadá- Ceará. Revista Brasileira de Plantas Medicinais. 17(3), 1516-0572, 2015.

Pereira, J. B. A.; Rodrigues, M. M.; Morais, I. R.; Vieira, C. R. S.; Sampaio, J. P. M.; Moura, M. G.; Damasceno, M. F. M.; Silva, J. N.; Calou, I. B. F.; Deus, F. A.; Peron, A.P.; Abreu, M. C.; Militão, G. C. G.; Ferreira, P. M. P. O papel terapêutico do Programa Farmácia Viva e das plantas medicinais no centro-sul piauiense. Revista Brasileira de Plantas Medicinais, 17(4), 1516-0572, 2015.

Posey, D. A. Introdução: Etnobiologia, teoria e pratica. In: Ribeiro, D. Suma Etnológica Brasileira. Petrópolis: Vozes; FINEP, p. 15-25, 1986.

Ribeiro, S. S. L. Estudo etnobotânico de plantas medicinais em uma área de Caatinga na Paraíba. 45f. Trabalho de Conclusão de Curso (Especialização - Gestão em Recursos Ambientais do Semiárido - GRAS) Instituto Federal de Educação Tecnológica da Paraíba, IFPB, Picuí. 2018.

Ruzza, D. A. C.; Göttert, V.; Rossi, A. A. B.; Dardengo, J. F. E.; Silva, I. V. Levantamento etnobotânico no município de alta floresta, Mato Grosso, Brasil. Enciclopédia Biosfera. 10(18), 2317-2606, 2014.

Schultes, R. E.; Reis, S. V. (eds). Ethnobotany: evolution of a discipline. Cambridge, Timber Press, 1995, p.414.

Silva, C. G.; Marinho, M. G. V.; Lucena, M. F. A.; Costa, J. G.M. Levantamento etnobotânico de plantas medicinais em área de Caatinga na comunidade do Sítio Nazaré, município de Milagres, Ceará, Brasil.Revista Brasileira de Plantas Medicinais. 17(1),1516-0572, 2015.

Soares, F.P.; Fraga, A.F.1.; Neves, J.P.O.; Romero, N.R.; BANDEIRA, M.A.M. Estudo etnofarmacológico e etnobotânico de Himatanthusdrasticus (Mart.) Plumel (janaguba). Revista Brasileira de Plantas Medicinais. 17(4), 1983-084X, 2015.

Souza, B. I.; Artigas, R. C.; LIMA, E. R. V. Caatinga e desertificação. Mercator. 14(1), 1984-2201, 2015.

Species Link- Disponível em: http://www.splink.org.br. Acesso em: 05 dezembro de 2020.

The Plant List. Disponível em: http://www.theplantlist.org. Acesso em: 05 de dezembro de 2020. 